\title{
FEATURE VECTOR CONSTRUCTION METHOD FOR IRIS RECOGNITION
}

\author{
G. Odinokikh ${ }^{\mathrm{a}, \mathrm{d}}$, A. Fartukov ${ }^{\mathrm{a}}$, M. Korobkin ${ }^{\mathrm{c}}$, J. Yoo $^{\mathrm{b}}$ \\ ${ }^{a}$ Samsung R\&D Institute Russia, 127018, Dvintsev st. 12, Moscow, Russian Federation - (g.odinokikh, a.fartukov)@ samsung.com \\ ${ }^{\mathrm{b}}$ Samsung Electronics Co. Ltd., 457-4 Maetan 3-dong, Suwon, South Korea - juwoan.yoo@ samsung.com \\ ${ }^{c}$ MIEE, 124498, Shokin sq., Zelenograd, Russia - mikhail.korobkin@ hotmail.com \\ ${ }^{\mathrm{d}}$ Dorodnicyn Computing Centre of RAS, 119333, Vavilov st. 40, Moscow, Russia
}

Commission II, WG II/10

KEY WORDS: Iris recognition, Iris feature extraction, Biometrics, Mobile biometrics, Quantization, Encoding

\begin{abstract}
:
One of the basic stages of iris recognition pipeline is iris feature vector construction procedure. The procedure represents the extraction of iris texture information relevant to its subsequent comparison. Thorough investigation of feature vectors obtained from iris showed that not all the vector elements are equally relevant. There are two characteristics which determine the vector element utility: fragility and discriminability. Conventional iris feature extraction methods consider the concept of fragility as the feature vector instability without respect to the nature of such instability appearance. This work separates sources of the instability into natural and encodinginduced which helps deeply investigate each source of instability independently. According to the separation concept, a novel approach of iris feature vector construction is proposed. The approach consists of two steps: iris feature extraction using Gabor filtering with optimal parameters and quantization with separated preliminary optimized fragility thresholds. The proposed method has been tested on two different datasets of iris images captured under changing environmental conditions. The testing results show that the proposed method surpasses all the methods considered as a prior art by recognition accuracy on both datasets.
\end{abstract}

\section{INTRODUCTION}

Recent years biometric technologies increasingly find their applications in many different areas. They are in widespread use particularly in access control systems field. Biometric authentication is considered as a candidate to replace conventional schemes that use keys, smart cards, personal identification numbers etc. The growth of interest in biometric technologies is associated mainly with security requirements strengthening of the system and their usability. Iris recognition has several advantages over other biometric technologies (Daugman, 2004, Chowhan et al., 2008, Bhattacharya and Mali, 2013, Gankin et al., 2014). Iris structure is persistent, well-expressed and highly informative biometric trait which is almost unchangeable throughout entire life. All the mentioned features of iris ensure achievement of higher recognition accuracy and reliability of iris-based biometric system. It makes iris recognition one of the most preferable technologies to be used in security systems and their applications.

One of the basic stages of iris recognition pipeline is the feature vector construction procedure. Basically, the procedure should have two important properties: saliency and suitability. Feature vector should contain distinctive information about iris texture and, at the same time, feature vector can be easily constructed, stored in compact way, and be suitable for subsequent matching (Jain et al., 2004).

Thorough investigation of feature vectors obtained from iris showed that not all the vector elements are equally relevant (Hollingsworth et al., 2009, Gneushev et al., 2015). There are two characteristics which determine the element utility: fragility and discriminability (Proença, 2015). The first one shows its reliability to natural and artificial variations of environment (illumination, sensor noise, etc.) as well as to processes occur within iris (pupil dilation/contraction mainly). The second characteristic reflects correlations in value across whole population. Thus, the feature selection procedure aims to pick the values which are both stable and discriminative enough, allowing iris recognition system work under various conditions while maintaining uniqueness property.

\section{FEATURE VECTOR CONSTRUCTION}

The procedure of iris feature vector construction is applied on normalized iris image (Figure 1). In this particular work both iris and pupil borders are considered as having circular shape. All the images in datasets used for testing have been preliminary marked by expert to extract information about iris/pupil centers and radii. This information is used further to obtain image of the normalized iris. Iris regions occluded by different noise factors such as eyelids, eyelashes, reflections and other, are commonly considered as information irrelevant for subsequent comparison and not used for the feature extraction. The entire procedure could be divided into two parts: extraction of iris texture features and their subsequent encoding as depicted in Figure 1.

A lots of the feature extraction methods, considering iris texture patterns on different detail levels, have been proposed (Bowyer et al., 2008). In this particular work the methods based on complex Gabor filtering are taken as a basis. Gabor-based methods are among the most accurate and robust and therefore used in many successful commercial solutions (Daugman, 2004). The feature encoding part is needed especially for raising of representation stability and matching process speed up. One of the most common encoding approaches, among the researchers, is a quantization of feature vector values. Despite the quantization is capable to remove irrelevant information it also could reduce one which is useful, thereby destabilize feature vector value or even make it 


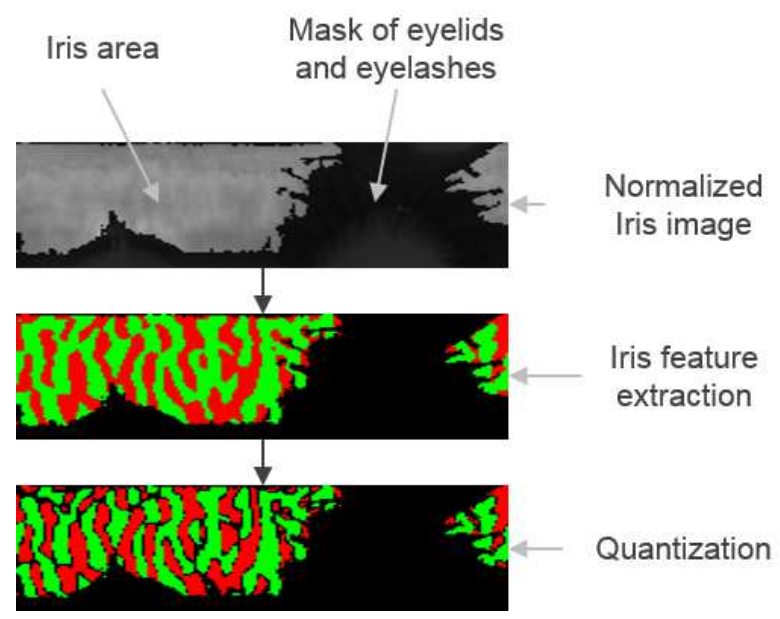

Figure 1. Feature vector construction procedure

fragile. But the method still has one important advantage leaving it so popular in use: a comparison of the quantized values is a bitwise operation which is fast itself.

All the state-of-art methods consider the concept of fragility as the feature vector instability without respect to the nature of such instability appearance. This work separates sources of the instability into natural and encoding-induced which helps deeply investigate the appearance. The work is mainly focused on encoding-induced fragility of the features obtained after complex Gabor filters applying.

A novel approach of iris feature vector construction is presented in this work. The approach consists of two steps: iris feature extraction using Gabor filtering with optimal parameters and quantization with separate preliminary optimized fragility thresholds.

\subsection{Iris feature extraction}

One of the of the feature extraction methods, used in many successful commercial iris recognition systems, is based on extraction of a quantized phase from a response of complex Gabor filters applied to an image. The method was first proposed in work (Daugman, 1993) and has since been subjected to various modifications (Si et al., 2012, Thornton et al., 2007). All of the related methods use either several filters with octave frequency increase or single filter with pre-defined parameters. The main advantage of Gabor-based method applying in this case is in its ability to construct band-pass filter with adjustable parameters. This property allows to set up a priori characteristics of analyzed object in a frequency domain. Under non-ideal conditions with a presence of correlated noise, caused by low-frequency difference in luminance, a higher recognition quality could be achieved with setting up the fine band-pass filter through optimization of its parameters.

Moreover, conventionally, complex Gabor filters are considered as whole but from fragility point of view it is beneficial to treat its real $(\mathrm{Re})$ and imaginary $(\mathrm{Im})$ parts separately. It allows to consider encoding as a way to obtain zero-crossing representation (Lee et al., 2013). Indeed, the previously introduced separation helps to find fragility thresholds (quantization thresholds) for each part independently (Figure 2).

In this work the single-filter approach with pre-optimized parameters, different for Re and Im parts, is proposed. The following parameters of Gabor kernel were chosen for optimization: wavelength $\lambda$, standard deviation $\sigma$ and spatial aspect ratio of the envelope $\gamma$ respectively. Many experiments conducted by us and other researchers showed that the most significant features of iris are orthogonal to its radial direction, thus $\theta=0$ is set up. An equal error rate (EER) value expressed in fractions is chosen as the objective function for optimization. The ERR denotes error rate corresponding to the threshold $t$ for which false match rate (FMR) is equal to false non-match rate $(F N M R)$ : $F M R(t)=\operatorname{FNMR}(t)$. Choosing of EER as the objective function allows to estimate performance of a recognition system regardless to pre-defined similarity threshold. Nelder-Mead direct search method (Nelder and Mead, 1965) is used for the optimization. The method proved itself well in optimization problem solving, in particular in case of presence of plateau regions and saddle points, due to its ability to of irregular simplex construction. Optimization and final testing are performed on CASIA-IrisV3-Lamp (Tan, 2010) dataset modeling changes in illumination condition during iris image capturing. The whole dataset has been split for train and test sets as $0.6 / 0.4$ for optimization and testing respectively.

In order to compare the performance, the testing is also performed for the octave frequency increase approach described above. The same subsequent quantization scheme, described in (Daugman, 1993), is used for both methods. Hamming distance (HD) is chosen as a conventional similarity measure for two iris feature vectors. The results are present in Table 1. The EER and also d' values are used for the evaluation. The d' metric is a mesure of general seperability between genuine and impostor distributions, that grows as both distribtuions move apart and become more narrow. It tends to be more sensitive metric than EER when distributions have a small intersection area.

\begin{tabular}{|l|c|c|}
\hline Method & EER & d' \\
\hline Octave frequency increase & 0.0406 & 3.61 \\
Proposed & 0.0373 & 3.73 \\
\hline
\end{tabular}

Table 1. Recognition accuracy evaluation results for different iris feature extraction methods

The results in Table 1 show that the approach with optimized kernel for each Re and Im parts outperforms conventional method using several filters with octave frequency increase.

\subsection{Quantization}

A quantization of extracted signal phase is the final step of iris feature vector construction procedure (Figure 1). Initially, it's performed depending on sign of the phase (Daugman, 1993) and all the elements are used for comparison. Further, in work (Hollingsworth et al., 2009) was shown that not all quantized feature vector elements are equally relevant and introduced a concept of fragility. Fragility, in this particular case, means inconsistency of information stored in two or more feature vectors of the same iris. Inconsistent elements could be determined either from several or single frame. This work is focused on the singleframe approach. The most of the state-of-art works (Lee et al., 2013, Hollingsworth et al., 2009) use constant pre-defined threshold value to classify vector elements into fragile and non-fragile, while the threshold is the same for both Re and Im parts.

In this work an adaptive method for fragility thresholds determination on a single frame is proposed. The thresholds are different for Re and Im parts and independent to each other (Figure 2). 
The algorithm of adaptive fragility threshold determination uses reference threshold value $T_{R}$ preliminary obtained through optimization and could be described as follows:

1. Values in feature vector are sorted as $F V=\{\min . . \max \}$

2. Target fragility threshold is determined as $T_{F}=F V\left[T_{R} *\right.$ $L]$, where $L$ is a size of the feature vector (Figure 3)

In this work the thresholds $T_{R}(R e)$ and $T_{R}(\operatorname{Im})$ are preoptimized independently on the training set using grid search.
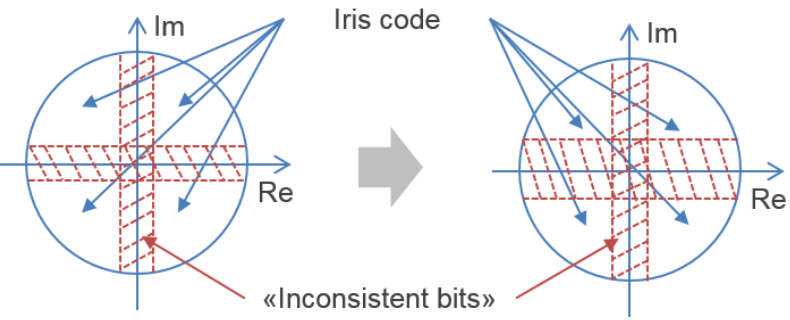

Figure 2. Different fragility thresholds for Re and Im parts

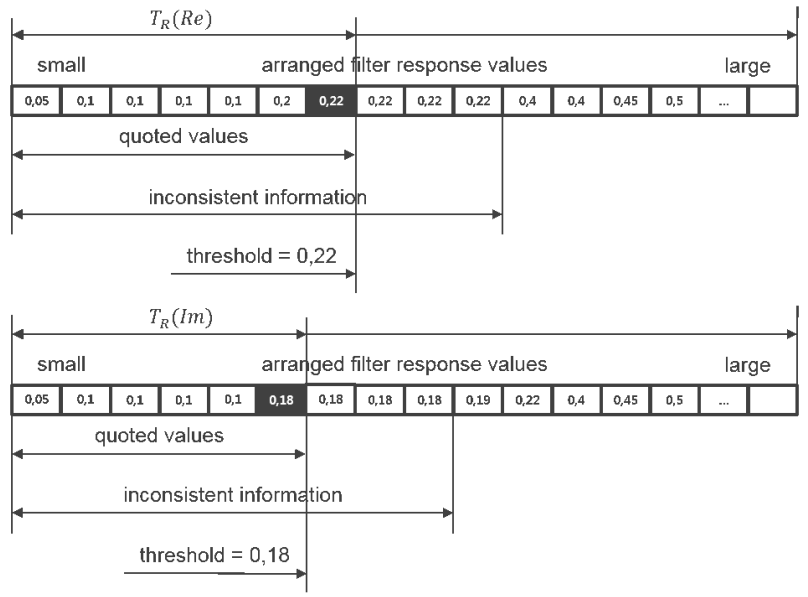

Figure 3. Fragility thresholds determination

\section{EXPERIMENTAL RESULTS}

\subsection{Datasets description}

Proposed feature extraction pipeline, including feature extraction and subsequent quantization, is tested on two different datasets of iris images. One of them CASIA-IrisV3-Lamp is publicly available and contains images captured under changing illumination level using hand-held iris sensor (Tan, 2010). Another used dataset has been collected privately using a hand-held mobile device as well but under highly changing environmental conditions. A specification of the dataset is present in Table 2. Some of the image examples of mobile dataset are present in Figure 4. Parameters of Gabor filter as well as reference fragility thresholds $\left(T_{R}\right)$ are pre-optimized for each dataset separately.

\subsection{Testing results}

In order to evaluate performance of the proposed quantization method, it has been compared with prior art method

\begin{tabular}{|l|c|c|}
\hline Dataset & CASIA & Mobile \\
\hline Subjects & 411 & 286 \\
Classes & 819 & 566 \\
Race & Asian & Asian \& Caucas. \\
Eyes on image & one eye & one eye \\
Capturing distance range & $15 \div 25(\mathrm{~cm})$ & $15 \div 35(\mathrm{~cm})$ \\
Camera resolution & $640 \times 480$ & $1280 \times 720$ \\
\hline
\end{tabular}

Table 2. Mobile dataset specification
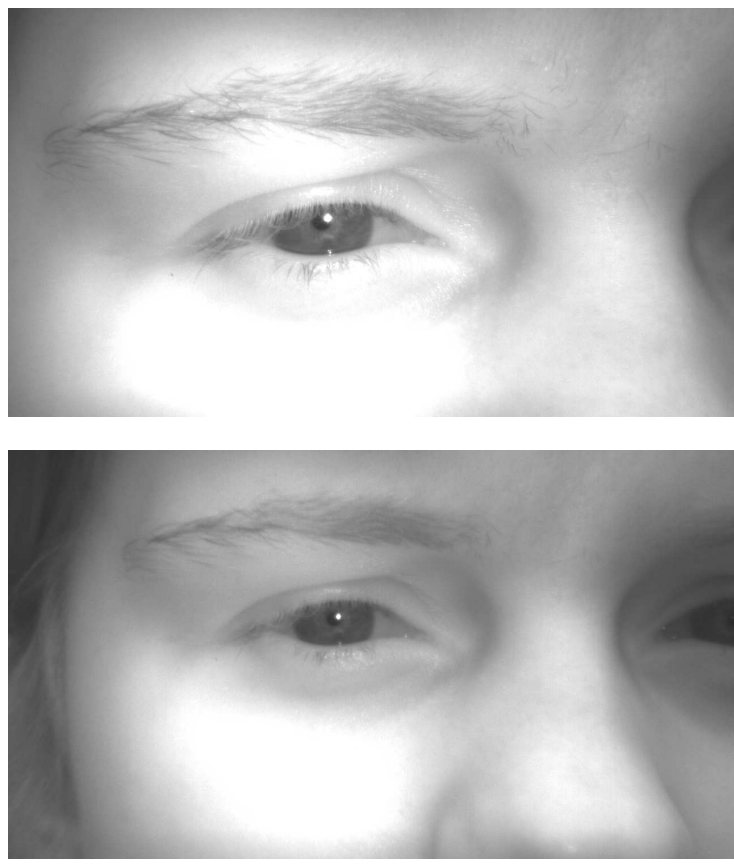

Figure 4. Mobile dataset image examples

(Hollingsworth et al., 2009). The EER and d' values are chosen for the evaluation. Proposed feature extraction is chosen as a beseline method for comparison as showed the best results. The testing results are present in Table 3.

\begin{tabular}{|l|c|c|c|c|}
\hline Dataset & \multicolumn{2}{|c|}{ CASIA } & \multicolumn{2}{c|}{ Mobile } \\
\hline & EER & d' & EER & d' \\
Baseline & 0.0373 & 3.73 & 0.0048 & 7.62 \\
Hollingsworth & 0.0430 & 3.60 & 0.0043 & 7.77 \\
Proposed & 0.0370 & 3.85 & 0.0040 & 8.01 \\
\hline
\end{tabular}

Table 3. Quantization methods comparision

The results (Table 3) showed that proposed method surpasses all the methods considered as a prior art by recognition accuracy on both datasets.

\section{CONSLUSIONS}

A novel approach of iris feature vector construction is proposed in this work. The approach consists of two steps: iris feature extraction using Gabor filtering with optimal parameters and quantization with separate preliminary optimized fragility thresholds (Figure 1). The proposed method is compared with two prior art methods on two different datasets of iris images and showed its advantage and applicability not only for standalone but for mobile iris biometric authentication systems as well. 


\section{REFERENCES}

Bhattacharya, V. and Mali, K., 2013. Iris as a biometric feature: Application, recognition, advantages \& shortcomings. International Journal of Advanced Research 3, no. 6, pp. 1410-1415.

Bowyer, K. W., Hollingsworth, K. and Flynn, P. J., 2008. Image understanding for iris biometrics: A survey. Computer vision and image understanding 110(2), pp. 281-307.

Chowhan, S., Cocsit, L. and Shinde, G., 2008. Iris biometrics recognition application in security management. In: Proceedings of Image and Signal Processing Conference (CISP), Sanya, Hainan, China, Vol. 1, pp. 661-665.

Daugman, J., 1993. High confidence visual recognition of persons by a test of statistical independence. IEEE Transactions on Pattern Analysis and Machine Intelligence 15, pp. 1148-1161.

Daugman, J., 2004. How iris recognition works. IEEE Transactions on Circuits and Systems for Video Technology 14(1), pp. 2130 .

Gankin, K., Gneushev, A. and Matveev, I., 2014. Iris image segmentation based on approximate methods with subsequent refinements. Journal of Computer and Systems Sciences International 53(2), pp. 224-238.

Gneushev, A., Kovkov, D., Matveev, I. and Novik, V., 2015. Optimizing the selection of a biometric template from a sequence. Journal of Computer and Systems Sciences International 54(3), pp. 399-405.

Hollingsworth, K. P., Bowyer, K. W. and Flynn, P. J., 2009. The best bits in an iris code. IEEE Transactions on Pattern Analysis and Machine Intelligence 31(6), pp. 964-973.

Jain, A. K., Ross, A. and Prabhakar, S., 2004. An introduction to biometric recognition. IEEE Trans. Cir. and Sys. for Video Technol. 14(1), pp. 4-20.

Lee, Y., Micheals, R., Filliben, J. and Phillips, J., 2013. Vasir: An open-source research platform for advanced iris recognition technologies. Journal of Research of the National Institute of Standards and Technology 118, pp. 244-247.

Nelder, J. A. and Mead, R., 1965. A simplex method for function minimization. Computer Journal 7, pp. 308-313.

Proença, H., 2015. Iris recognition: What is beyond bit fragility? IEEE Transactions on Information Forensics and Security 10(2), pp. 321-332.

Si, Y., Mei, J. and Gao, H., 2012. Novel approaches to improve robustness, accuracy and rapidity of iris recognition systems. IEEE Transactions on Industrial Informatics 8, pp. 110117.

Tan, T., 2010. CASIA-IrisV3. http://biometrics. idealtest.org/dbDetailForUser.do?id=3. [Online; accessed 15-January-2017].

Thornton, J., Savvides, M. and Kumar, V., 2007. An evaluation of iris pattern representations. IEEE International Conference on Biometrics, Theory, Applications and Systems 2, pp. 1-6. 